\title{
Comment on "A special attack on the multiparty quantum secret sharing of secure direct communication using single photons"
}

\author{
Cheng-An Yen ${ }^{\mathrm{a}}$, Shi-Jinn Horng ${ }^{\mathrm{a}}$, Hsi-Sheng Goan ${ }^{*, b, c}$, Tzong-Wann Kao ${ }^{\mathrm{d}}$ \\ ${ }^{a}$ Department of Computer Science and Information Engineering, National Taiwan University of Science and Technology, Taipei, Taiwan \\ ${ }^{b}$ Department of Physics and Center for Theoretical Sciences, National Taiwan University, Taipei 10617, Taiwan \\ ${ }^{c}$ Center for Quantum Science and Engineering, National Taiwan University, Taipei 10617, Taiwan \\ ${ }^{d}$ Department of Electronic Engineering, Technology and Science Institute of Northern Taiwan, Taipei, Taiwan
}

\section{Abstract}

In this comment, we show that the special attack [S.-J. Qin, F. Gao, Q.-Y. Wen, F.-C. Zhu, Opt. Commun. 281 (2008) 5472.], which claims to be able to obtain all the transmitted secret message bit values of the protocol of the multiparty quantum secret sharing of secure direct communication using single photons with random phase shift operations, fails. Furthermore, a class of similar attacks 'are also shown to fail to extract the secrete message.

Key words: Quantum secure direct communication, Quantum secret sharing, Quantum random phase shift operation त 'PACS: 03.67.Hk, 03.65.Ud, 03.67.Dd

In a recent paper, Qin et al. [1] pointed out that the HLLZ protocol [2] is vulnerable to a special participant attack strategy (hereafter called the QGWZ attack) where Alice's secret message can be extracted by any dishonest agent without any help of the other agents. However, in this paper, we comment that 'some errors may exist in the QGWZ attack and thus it fails to attack HLLZ protocol successfully.

The six main stages of the HLLZ protocol are preparation phase, encryption phase, the first detection, encoding phase, recovery phase and the second detection[2]. In short, based on the linear property of the quantum random phase shift op'erations (RPSOs) or equivalently random single-qubit rotations along the $y$-axis, the encryption, encoding and recovering phase of this protocol can be expressed as the addition/subtraction of the rotation angles on each photon (qubit) at different stages:

$$
\hat{U}\left(\theta_{j}\right) \stackrel{\hat{U}\left(3 m_{j} \pi / 2\right)}{\Longrightarrow} \hat{U}\left(\theta_{j}+3 m_{j} \pi / 2\right) \stackrel{\hat{U}\left(-\theta_{j}\right)}{\Longrightarrow} \hat{U}\left(3 m_{j} \pi / 2\right) .
$$

Here $\hat{U}\left(\theta_{j}\right)$ represents a rotation on photon $j$ with $\theta_{j}=\theta_{B_{j}}+$ $\theta_{C_{j}}+\cdots+\theta_{Z_{j}}$, and $3 m_{j} \pi / 2$ is the rotation angle made by Alice on the photon $j$ according to the bit value $m_{j}=0$ or 1 of her secret message $\mathbf{m}$. The measurement action in the recovery phase by the agent (say Zach), to which Alice sent the photons with encoded message, reflects basically whether there is a rotation of an angle $\frac{3}{2} \pi$ on the photon (qubit) $j$ or not. If there is, the bit value of the message bit $j$ is $m_{j}=1$; otherwise, $m_{j}=0$.

Grasping the main steps of the HLLZ protocol, the scenario for an dishonest agent (say Charlie) in the QGWZ attack is to entangle the transmitted photons with prepared ancilla photons firstly, to escape from the detection secondly, and to attain the

\footnotetext{
${ }^{*}$ Corresponding author. Tel: +8862 3366 5163; Fax: +886223639984.

Email address: goan@phys.ntu.edu.tw (Hsi-Sheng Goan)
}

secret message without being discovered lastly [1]. However, the errors that existed in the Ref. [1] will doom Charlie to fail to extract the secret message even if he can escape from the detection. The first typo in Ref. [1] is in the last sentence of the first paragraph of step (E2) in the second column on page 5473. The correct sentence for that should read as "Then Charlie sends the fake sequence $T$ to Alice.”. In other words, the sequence that was sent out by Charlie is $T$ rather than $H$ stated in Ref. [1]. But this typo is very minor as it is obvious from the subsequent description in Ref. [1] that it is the $T$ sequence that was sent out.

The possible serious error is in the expression of Eq. (9) of Ref. [1] which was derived from Eq. (7) of Ref. [1] by a controlled-controlled- $\left(-i \sigma_{y}\right)$ operation (referred to as $C Y$ operation in [1]) with photons $H_{j}$ and $T_{j}$ being the control qubits and photon $S_{j}$ being the target qubit. The action of $C Y$ operation on computational basis states is given by $C Y|t\rangle|c 1\rangle|c 2\rangle=$ $\left(-i \sigma_{y}\right)^{c 1 \otimes c 2}|t\rangle|c 1\rangle|c 2\rangle$, where $c 1$ and $c 2$ represent the control qubits and $t$ stands for the target qubit on which the $-i \sigma_{y}$ operation acts. So the correct expression for Eq. (9) of Ref. [1] should read as

$\left|\Psi_{3}^{0}\right\rangle_{S_{j} H_{j} T j}=\frac{1}{2}(\cos \theta|0\rangle-\sin \theta|1\rangle)_{S_{j}}(|00\rangle+|11\rangle-|01\rangle+|10\rangle)_{H_{j} T_{j}}$.

Comparing Eq. (2) above with Eq. (10) of Ref. [1], one can find that the two $H_{j} T_{j}$ photon states in those two equations are identical rather than orthogonal. The statement that the two states of photons $H_{j} T_{j}$ are orthogonal in Ref. [1] comes from comparing the incorrect Eq. (9) of Ref. [1] with Eq. (10) of Ref. [1]. Therefore, Charlie cannot, by measuring photons $H_{j} T_{j}$, distinguish the state $\left|\Psi_{3}^{0}\right\rangle_{S_{j} H_{j} T j}$ of the correct Eq. (2) from the state $\left|\Psi_{3}^{1}\right\rangle_{S_{j} H_{j} T j}$ of Eq. (10) of Ref. [1], and thus cannot extract Al- 
ice's secret bit value in practice. As a result, the QGWZ attack fails to extract Alice's secret message bits from the HLLZ protocol.

The main idea of the QGWZ attack seems (i) to use an entanglement operation to entangle each of the photon in sequence $S$ with prepared ancilla photons to escape the first detection of the HLLZ protocol, (ii) to use the inverse entanglement operation to separate the entangled states back to separable state to obtain the secret message and to escape the second detection of the HLLZ protocol. The particular entanglement operation in the Ref. [1] is $C Y$ and we have shown that it fails to extract the secret message. One may wonder whether Charlie can extract the secret with other operations instead of $C Y$ in this QGWZlike attack. In fact, we show below that Charlie will still fail to extract the secret even if he can apply other entanglement operations in the QGWZ-like attacks. Without loss of generality, suppose the photon $j$ in the $S$ sequence sent out by the last agent, Zach, is in the state

$$
|\chi\rangle_{S_{j}}=\cos \theta_{j}|0\rangle_{S_{j}}+\sin \theta_{j}|1\rangle_{S_{j}}
$$

where $\theta_{j}=\theta_{B_{j}}+\theta_{C_{j}}+\cdots+\theta_{Z_{j}}$. In this way, Charlie may prepare his ancilla qubits sequence $A$ each in the state $|\varepsilon\rangle_{A_{j}}$, and apply a particular kind of entanglement operation $\hat{E}$ on $|\chi\rangle_{S_{j}}$ such that

$$
\hat{E}\left(|\varepsilon\rangle_{A_{j}} \otimes|\chi\rangle_{S_{j}}\right)=\alpha|\varepsilon\rangle_{A_{j}}|\chi\rangle_{S_{j}}+\beta\left|\varepsilon^{\perp}\right\rangle_{A_{j}}\left[\hat{U}\left(\theta^{\prime}\right)|\chi\rangle\right]_{S_{j}},
$$

where $|\alpha|^{2}+|\beta|^{2}=1$ and $\left\langle\varepsilon \mid \varepsilon^{\perp}\right\rangle_{A_{j}}=0$, and $U\left(\theta^{\prime}\right)$ is the rotation operation along the $y$ axis defined in (1). After these operations, Charlie sends sequence $S$ to Alice, and he may escape from Alice's first detection by telling the value of his rotation angle of either $\theta_{c}$ or $\left(\theta_{c}+\theta^{\prime}\right)$. That is, when Alice selects the photon $j$ to check the security of the quantum channel used, Charlie can first measure the corresponding ancilla qubit $A_{j}$ in the $\left\{|\varepsilon\rangle,\left|\varepsilon^{\perp}\right\rangle\right\}$ basis. He then announces his rotation angle of either $\theta_{c}$ or $\left(\theta_{c}+\theta^{\prime}\right)$ when the measured result is in $|\varepsilon\rangle$ or in $\left|\varepsilon^{\perp}\right\rangle$, respectively. As a result, Alice will not discover Charlie's dishonest action during her first detection step. From this point of view, the QGWZ attack of Ref. [1] is just a simplified special case of above description where Charlie's $\hat{E}$ is the controlledcontrolled- $\left(-i \sigma_{y}\right)$ operation and the rotated phase shift angle $\theta^{\prime}$ in Eq. (4) is $-\frac{3}{2} \pi$. In short, Charlie hence can escape from the first detection in the HLLZ protocol with this QGWZ-like attack.

After that, in the HLLZ protocol, Alice will encode her secret message bit value on each of the remaining photons in sequence $S$ with a rotation operation along the $y$-axis by an angle of $\frac{3}{2} \pi$ if the bit value is 1 . Otherwise, no operation is performed if the bit value is 0 . This message-encoding operation can be expressed as

$$
\hat{U}(3 \pi / 2)=-i \sigma_{y}=|1\rangle\langle 0|-| 0\rangle\langle 1| .
$$

When it is applied to the state of Eq. (4), let us consider the following transformation.

$$
\begin{aligned}
\hat{U}(3 \pi / 2)|\chi\rangle_{S_{j}} & =\cos \theta_{j}|1\rangle_{S_{j}}-\sin \theta_{j}|0\rangle_{S_{j}} \\
& =\cos \left(\theta_{j}-\frac{3}{2} \pi\right)|0\rangle_{S_{j}}+\sin \left(\theta_{j}-\frac{3}{2} \pi\right)|1\rangle_{S_{j}} \\
& =\cos \theta_{j}^{\prime}|0\rangle_{S_{j}}+\sin \theta_{j}^{\prime}|1\rangle_{S_{j}} \\
& \left.\equiv \chi^{U}\right\rangle_{S_{j}}
\end{aligned}
$$

where $\theta_{j}^{\prime}=\theta_{j}-\frac{3}{2} \pi$. Since $\hat{U}(3 \pi / 2)$ of Eq. (5) commutes with $\hat{U}\left(\theta^{\prime}\right)$ in Eq. (4), the resultant state becomes

$$
\alpha|\varepsilon\rangle_{A_{j}}\left|\chi^{U}\right\rangle_{S_{j}}+\beta\left|\varepsilon^{\perp}\right\rangle_{A_{j}}\left[\hat{U}\left(\theta^{\prime}\right)\left|\chi^{U}\right\rangle\right]_{S_{j}}
$$

if Alice's secret message bit value is 1 . Otherwise, if Alice's secret message bit value is 0 , the resultant state remains in the state of Eq. (4).

After intercepting the transmitted photon again, Charlie will apply the inverse entanglement operation $\hat{E}^{-1}$ to separate the entanglement between photon $A_{j}$ and $S_{j}$ in the QGWZ-like attack. He then will try to figure out Alice's secret message by making an measurement on his ancilla qubits in the orthonormal basis of $\left\{|\varepsilon\rangle,\left|\varepsilon^{\perp}\right\rangle\right\}$. Since $\hat{E}^{-1} \hat{E}=I$, if Charlie applies the operator $\hat{E}^{-1}$ to Eq. (4), the state of the photons $A_{j}$ and $S_{j}$ will become

$$
\hat{E}^{-1}\left(\alpha|\varepsilon\rangle_{A_{j}}|\chi\rangle_{S_{j}}+\beta\left|\varepsilon^{\perp}\right\rangle_{A_{j}}\left[\hat{U}\left(\theta^{\prime}\right)|\chi\rangle\right]_{S_{j}}\right)=|\varepsilon\rangle_{A_{j}} \otimes|\chi\rangle_{S_{j}} .
$$

It follows that if the operator $\hat{E}^{-1}$ is applied to Eq. (7), it will become

$$
\hat{E}^{-1}\left(\alpha|\varepsilon\rangle_{A_{j}}\left|\chi^{U}\right\rangle_{S_{j}}+\beta\left|\varepsilon^{\perp}\right\rangle_{A_{j}}\left[\hat{U}\left(\theta^{\prime}\right)\left|\chi^{U}\right\rangle\right]_{S_{j}}\right)=|\varepsilon\rangle_{A_{j}} \otimes\left|\chi^{U}\right\rangle_{S_{j}} .
$$

Since the photon state $|\varepsilon\rangle_{A_{j}}$ is the same in both Eq. (8) and Eq. (9), it is obvious that Charlie cannot distinguish whether the state of photon $S_{j}$ has been rotated by an angle of $\frac{3}{2} \pi$ or not from the measurement result of the ancilla photons $A_{j}$. Thus Charlie cannot extract the transmitted secret bits even if he can apply the entanglement operations defined in Eq. (4) in the QGWZ-like attacks.

In summary, the QGWZ attack as well as the QGWZ-like attacks which apply entangled operations other than the $C Y$ operation fail to obtain the transmitted secret message bits of the HLLZ protocol.

\section{Acknowledgments}

H.S.G. would like to acknowledge support from the National Science Council, Taiwan, under Grant No. 97-2112-M002-012-MY3, support from the Frontier and Innovative Research Program of the National Taiwan University under Grant Nos. 97R0066-65 and 97R0066-67, and support from the focus group program of the National Center for Theoretical Sciences, Taiwan. C.A.Y. and S.J.H. would like to acknowledge support from the National Science Council, Taiwan, under Grants Nos. 97-2221-E-239-022- and 95-2221-E-011-032-MY3.

\section{References}

[1] S.-J. Qin, F. Gao, Q.-Y. Wen, F.-C. Zhu, Opt. Commun. 281 (2008) 5472

[2] L.-F. Han, Y.-M. Liu, J. Liu, Z.-J. Zhang, Opt. Commun. 281 (2008) 2690. 